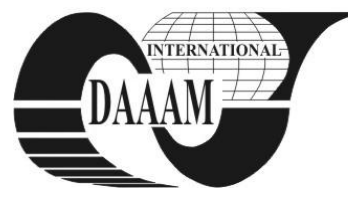

\title{
A NEW APPROACH ON THE OFFSET OF MAGNETIC MICROSENSORS
}

\author{
CARUNTU, G[eorge]; TAMAS, R[azvan] \& DRAGOMIRESCU, O[vidiu]
}

\begin{abstract}
One of the essential parameters in setting up the performances of the measurement systems that uses Hall microsensors is the magnetic offset of such devices.

This paper presents the structure, the operating conditions and the main characteristics of double drain magnetotransistors.

Numerical simulations shows the fact that an adequate choice of the device geometry and material features are key parameters for obtaining high performances of the devices. In the final part are also presented and described original electrical diagrams of some transducers which contain such sensors.
\end{abstract}

Key words: double-drain magnetotransistor, offset collector current, offset equivalent magnetic induction, carrier mobility.

\section{INTRODUCTION}

This paper presents a number of research results regarding analysis and optimization of double drain magnetotransistor structures.

The offset equivalent magnetic induction is usually defined for conventional Hall devices on experimental basis, without any studies regarding optimization or at least reduction of each value.

In this paper, based on adequate models, we established the offset-equivalent magnetic induction for double drain magnetotransistor.

By using numerical simulation, the values of the offsetequivalent magnetic induction for the devices analysed are compared. Moreover, it is also emphasised the way in which the geometry and the material features choose allows obtaining sensors with higher performances.

The research might be further continued in order to develop new technologies and structures for magnetic microsensors, capable to obtain even smaller values for the offset-equivalent magnetic induction.

\section{THE DOUBLE-DRAIN MOSFET}

The double - drain MOS device is obtained from a MOSFET structure where its conventional drain region is replaced by two adjacent drain regions, as shown in figure 1 .

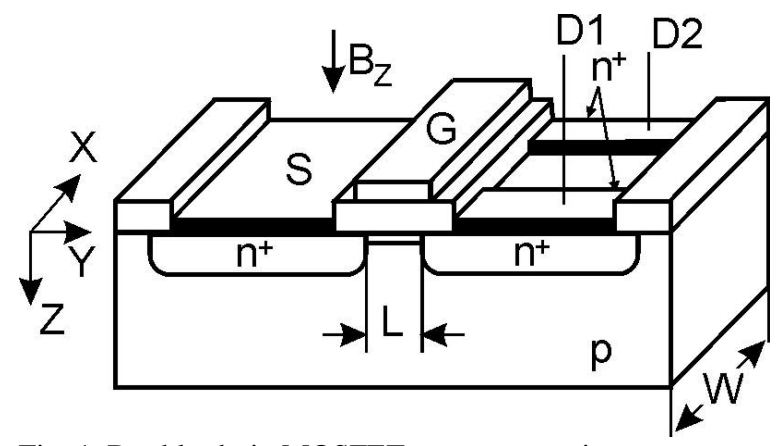

Fig. 1. Double-drain MOSFET magnetotransistor
Consequently, the total channel current is shared between the two drain regions (Nathan, 1985).

Two additional strongly doped $\left(n^{+}\right)$regions, are used as contacts for sensing the Hall voltage. The channel length is $L$, its width is $W$.

Since the bias is in the linear region, a continuous channel of approximately constant thickness is obtained, which can be assimilated with a Hall plate.

The deflection of current lines appears under the action of a magnetic field $B_{\perp}$, perpendicular to the device surface. The carrier deflection causes a discrepancy between two drain currents: $\Delta I_{D}=I_{D}(B)-I_{D}(0)$. Since the output signal of the double-drain magnetotransistors consists of the current variation between its terminals, this device operates in the Hall current mode. Using the features of dual Hall devices the Hall current expression, results (Popovic, 1991)

$$
\Delta I_{D}=\frac{I_{H}}{2}=\frac{1}{2} \mu_{H_{C h}} \cdot \frac{L}{W} \cdot G \cdot I_{D} \cdot B_{\perp}
$$

where $\mu_{H_{C h}}$ denotes the carriers Hall mobility, $G$ is the geometrical correction factor and $I_{D}$ is the drain current. The supply-current-related sensitivity of the devices is defined by:

$$
S_{I}=\frac{1}{I_{D}} \cdot\left|\frac{\Delta I_{D}}{B_{\perp}}\right|=\frac{1}{2} \mu_{H_{C h}} \cdot \frac{L}{W} G
$$

The sensor response is expressed by (Căruntu, 2007):

$$
h(B)=\frac{\Delta I_{D}}{\left(I_{D 1}+I_{D 2}\right)_{B=0}}=\frac{1}{2} \mu_{H_{C h}} \frac{L}{W} \cdot G \cdot B_{\perp}
$$

and it is linear for induction values which satisfy the condition: $\mu_{H}^{2} \cdot B_{\perp}^{2} \ll<1$.

\section{THE OFFSET-EQUIVALENT MAGNETIC INDUCTION}

In the absence of the magnetic field, the difference between the two drain currents is the offset collector current:

$$
\Delta I_{D_{\text {off }}}=I_{D 1}(0)-I_{D 2}(0)
$$

This offset current appears due to the imperfections specific to the manufacturing process: the contact non-linearity, the non-uniformity of the thickness and of the epitaxial layer doping, the presence of some mechanical stresses combined with the piezo-effect.

In order to describe the error due to the offset, the magnetic induction which produce the imbalance $\Delta I_{C}=\Delta I_{C_{\text {off }}}$ has to be determined.

Using relation (2), the offset equivalent magnetic induction is expressed by: 


$$
B_{\text {off }}=\frac{\Delta I_{D_{\text {off }}}}{S_{I} I_{D}}=\frac{2}{\mu_{H n}} \cdot \frac{\Delta I_{D_{\text {off }}}}{I_{D}} \cdot\left(G \frac{L}{W}\right)^{-1}
$$

Simulations have been performed considering $\Delta I_{D_{\text {off }}}=0.10 \mu \mathrm{A}$ and assuming that the low magnetic field condition is achieved. In figure 2 is presented the dependence of $B_{\text {off }}$ on $I_{D}$ for three magnetotransistors with the same geometry $W / L=0.5$ realised from different materials:

$$
\begin{aligned}
& \text { MDD1: Si with } \mu_{H_{C h}}=0.07 m^{2} V^{-1} s^{-1} ; \\
& \text { MDD2: InP with } \mu_{H_{C h}}=0.23 m^{2} V^{-1} s^{-1} ; \\
& \text { MDD3: GaAs with } \mu_{H_{C h}}=0.43 m^{2} V^{-1} s^{-1}
\end{aligned}
$$

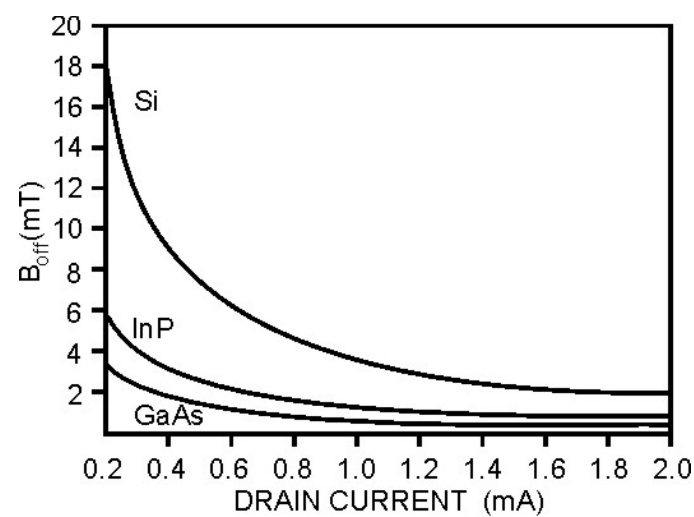

Fig. 2. The $B_{\text {off }}$ depending on the drain current for three devices of different materials

The geometry influence on $B_{\text {off }}$ is shown in figure 3 by simulating three magnetotransistors structures realised from silicon and having different $W / L$ ratios (Middelhoek,1989).

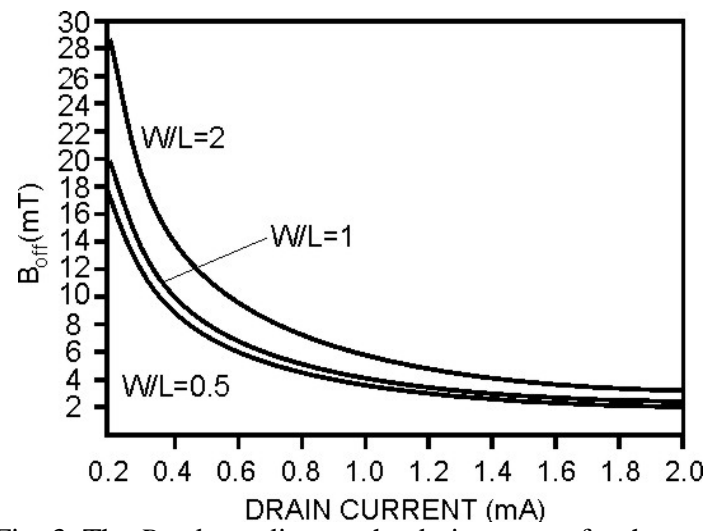

Fig. 3. The $B_{\text {off }}$ depending on the drain current for three devices of different geometry

$$
\begin{gathered}
\text { MDD1: } \quad W / L=0,5 ; G(L / W)=0.73 ; \\
\text { MDD2: } W / L=1 ; G(L / W)=0.67 ; \\
\text { MDD3: } W / L=2 ; G(L / W)=0.47
\end{gathered}
$$

Simulations have shown that if the width of the channel is maintained constant, $B_{\text {off }}$ increases as the channel length decreases. Therefore, the minimum values for the offset equivalent induction are obtained with the device which has $L=2 W$, and in the MDD3 device these values are $53.5 \%$ larger.

\section{CONCLUSIONS}

The analysis of the characteristics of the double drain magnetotransistor shows that the $W / L=0.5$ ratio is theoretically favourable to high performance regarding the offset equivalent magnetic induction.

It has to be noticed that by increasing the channel length we can decrease $B_{\text {off }}$ with $31 \%$ for a square structure $(W=L)$ and with $38 \%$ for $W=0.5 L$.

Also, substituting the silicon technology with other materials such as GaAs or InSb with high carrier mobility values insures higher performances for the developed sensors.

The offset equivalent magnetic induction lowers with the increase of carriers mobility, this increase being significant for collector currents of relatively low values.

Consequently, for the drain current $I_{D}=0.2 \mathrm{~mA}$, the offset equivalent magnetic induction value of the GaAs device decreases by $81.8 \%$ as compared to that of the silicon device. The optimal processing of sensors - provided signal impose their integration on the same chip with the amplifier circuit.

Figure 4 shows the electrical diagram of a transducer based on double-drain magnetotran-sistors (Panait, 2009).

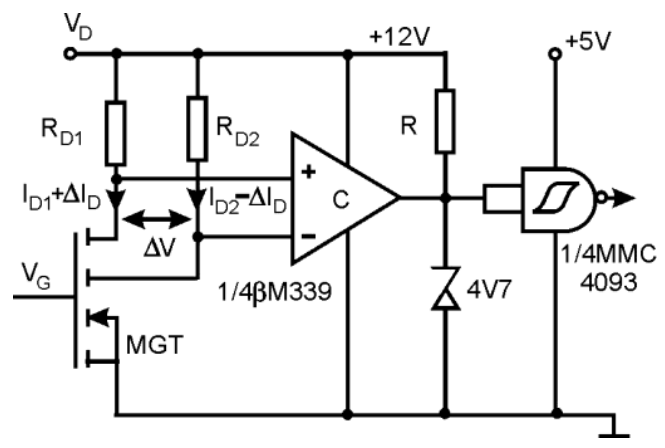

Fig. 4. The electrical diagram of transducer

In the double-drain MOSFET works in saturation the differential output voltage is:

$$
\Delta V_{D}=\mu_{H_{C h}} \frac{L}{W} G V_{R} B_{\perp}
$$

Although the magnetotransistors have a low magnetic sensitivity, very large signal-to-noise ratios are obtained, hence, a high magnetic induction resolution is resulting. This voltage is applied to a comparator with hysteresis, which acts as a comutator.

\section{REFERENCES}

Căruntu,G.(2007) The magnetic microsensors response, , Przeglad Elektrotechniczny, Nr2/2007,pp 33-77 ISSN17316106 R, Poland

Middelhoek, S.; Audet, S.A.(1989) Physics of Silicon Sensors, pp. 5.20-5.24,Academic Press,ISBN 0124950511, London

Nathan, A.; Huiser, A. M. J.\& Baltes, H. P.( 1985).Two Dimensional Numerical Moddeling of Magnetic Field Sensors in CMOS Technology, IEEE Trans. Electron Device ED-32 1212-19,ISSN 0018-9383

Panait, C.(2009). The Offset of Magnetic Microsensors, Proceedings of the ISTET'09,June 22-24, Germany, ISSN 0932-602, pp.92-95, Lübeck

Popovic, R.S. (1991). Hall Effect Devices, Magnetic Sensors and Characterization of Semiconductors, Adam Hilger, ISBN 0750300965, Bristol, England 